\title{
High-Throughput Large Volume SEM Workflow using Sparse Scanning and In- painting Algorithms Inspired by Compressive Sensing
}

\author{
Faysal Boughorbel ${ }^{1}$, Pavel Potocek ${ }^{1}$, Miloš Hovorka ${ }^{2}$, Libor Strakoš ${ }^{2}$, John Mitchels ${ }^{2}$, Tomáš Vystavěl ${ }^{2}$, \\ Patrick Trampert ${ }^{3}$, Ben Lich ${ }^{1}$, and Tim Dahmen ${ }^{3}$ \\ ${ }^{1}$ Thermo Fisher Scientific, Eindhoven, the Netherlands \\ ${ }^{2}$ Thermo Fisher Scientific, Brno, Czech Republic \\ ${ }^{3}$ DFKI Gmbh, Saarbrücken, Germany
}

We are presenting a new extension to our Cell and Tissue/Neurobiology large volume imaging workflow, with the goal of increasing acquisition speed by more than five times. Instead of scanning dense square-grid frames, in the conventional way, our approach is here to explore the use of sparse scanning and inpainting techniques inspired by Compressive Sensing (CS) [1]. Sparse samples are obtained by pseudo-random scan patterns, and reconstruction algorithms are used to recover the dense volume data. The goal is to recover 3D datasets with minimum loss of information. Techniques inspired by CS gained wide attention over the last decade and are now being used in various applications where sensor bandwidth is a limiting factor. They have been recently explored for SEM and STEM applications [2][3]. In the context of nano-scale cell biology volume acquisition, we expect these techniques to ultimately increase the imaging throughput by nearly an order of magnitude. We will discuss additional advantages of this approach, such as the low-dose imaging of sensitive specimens, and the good compatibility with backscatter electron imaging. A key enabler of any sparse scan application to EM is the accurate control of scan locations. It has been shown in [2] and in our own experiments that precise positioning of the beam at the planned sampling locations is essential for a good CS reconstruction. We have developed advanced minimum-path scanning strategies to address this issue. The scanning technique is illustrated in Fig. 1, where the left two images show a conventional raster scan at 300ns dwell visiting a random set of points with the compressive sensing reconstruction obtained from such scan strategy. The right two images of Fig. 1 show an example minimum-path scan pattern and a much improved reconstruction result from images acquired with this second method. In future work we will compare pseudo-random sparse sampling, in combination with a reconstruction algorithm based on CS-inspired in-painting, to conventional grid sampling of the same effective dose, in combination with a de-noising algorithm, also based on CS.

CS machine learning algorithms build patch-dictionaries, which are used as the building blocks for data representation [3]. During live acquisition runs, such dictionaries can be used to in-paint with high fidelity, the sparsely sampled datasets (Figure 2). We are implementing the new sparse scanning modules on SEM platforms, which also employ the Multi Energy Deconvolution SEM (MED-SEM) technology and Serial Block Face (SBF) imaging [4]. By incorporating CS, we will have an instrument allowing for both high-resolution isotropic imaging, and the fast acquisition of very large datasets (Figure 3).

References:

[1] E. J. Candès, International Congress of Mathematicians, 3 (2006), p. 1433.

[2] H. A. Anderson et al, IS\&T/SPIE Electronic Imaging, (2013), p. 86570C.

[3] A. Stevens et al, Microscopy, 63-1 (2014), p. 41.

[4] F. Boughorbel et al, Microsc. Microanal. 19.S2 (2013), p. 380. 

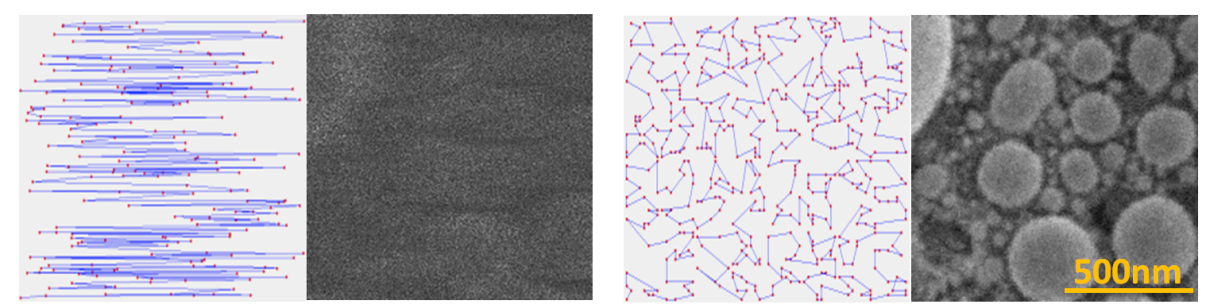

Figure 1. Scan pattern optimization for compressive sensing SEM acquisition and reconstruction: The sparsity of the acquired images is $20 \%$ (relative to full-grid dense sampling of the same area). The shown scan path diagrams contain simplified paths to explain the concept.

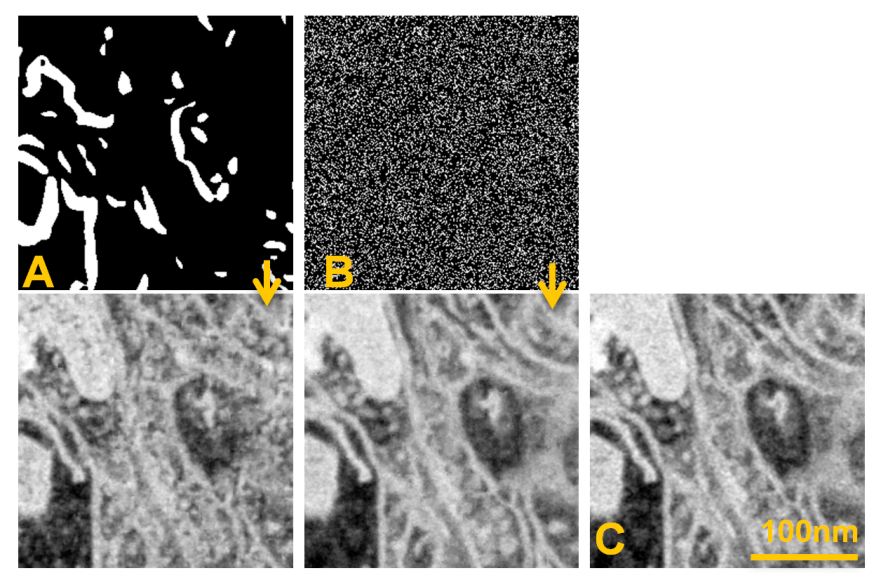

Figure 2. Adaptive and sparse acquisition strategies: In scheme (A) the bright regions $(20 \%$ of the image) are scanned with $1 \mu \mathrm{s}$ dwell, while the black regions are scanned at 100ns, denoised and recombined with the slow scan. In scheme (B) a random scan pattern is used (also $20 \%$ of data at $1 \mu \mathrm{s}$ dwell) then a CS in-painting is employed to fill the image. (C) is the full grid image at $1 \mu$ s dwell.

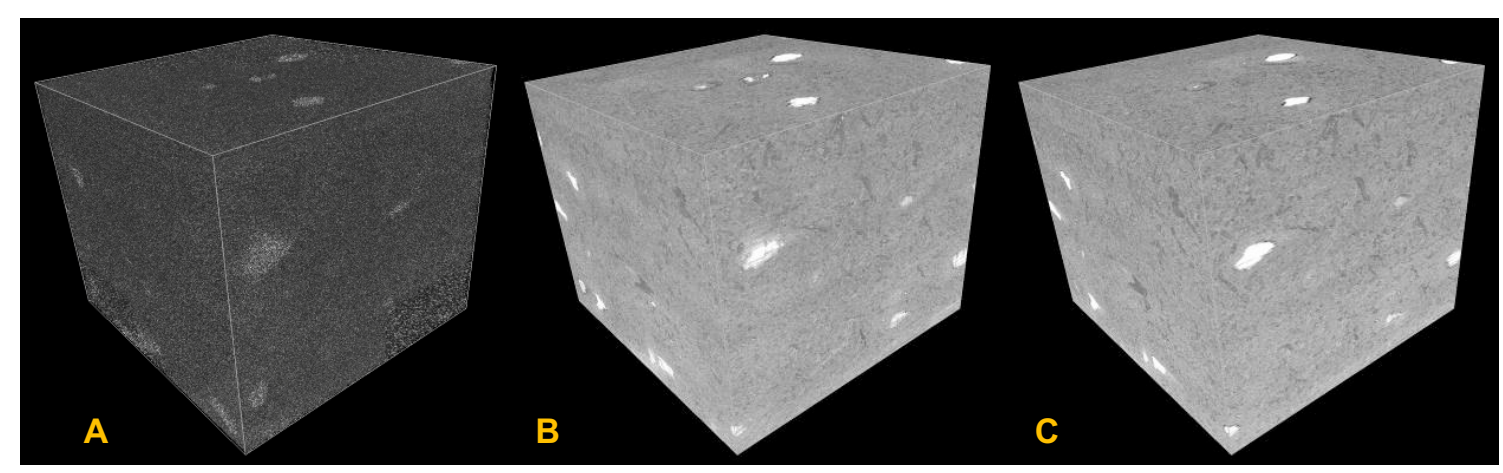

Figure 3. CS Large Volume acquisition: (A) is the volume acquired using SBF and sparse sampling, (B) the CS reconstruction, and $(\mathrm{C})$ the full-grid scanned volume $(100 \times 100 x 80 \mu \mathrm{m}$ volume; voxel size: 10x10x30nm; BSE imaging; dwell time: 1 $\mu$ s; sparsity of CS data: 20\% of full-grid samples). 